\title{
Body composition of preterm infants during infancy
}

D J Rawlings, R J Cooke, K McCormick, I J Griffin, K Faulkner, J C K Wells, J S Smith, S J Robinson

\begin{abstract}
Aims-To examine body composition in preterm infants.

Methods-Body composition was measured by dual energy $x$-ray absorptiometry (DEXA) at hospital discharge, term, 12 weeks, and at 6 and 12 months corrected age in 125 infants (birthweight $\leqslant 1750 \mathrm{~g}$, gestational age $\leqslant 34$ weeks).

Results-Body weight derived by DEXA accurately predicted that determined by conventional scales. In both sexes lean mass (LM), fat mass (FM), \%FM, bone area (BA), bone mineral mass (BMM), and bone mineral density (BMD) increased rapidly during the study; significant changes were detectable between discharge and term. At 12 months, LM, BA, and BMM, but not FM, \%FM, or BMD were greater in boys than in girls. Corrected for age, $L M$ was less than those of the reference term infant; FM and \%FM were similar; BMM was greater. Corrected for weight, $L M$ was similar to those of the reference infant, while the FM and $\%$ FM of study infants were slightly greater.

Conclusions-DEXA accurately measures body mass. Body composition in preterm boys and girls differs. Interpretation of DEXA values may depend on whether age or body weight are regarded as the appropriate reference.
\end{abstract}

(Arch Dis Child Fetal Neonatal Ed 1999;80:F188-F191)

Special Care Baby

Unit

Royal Victoria

Infirmary

Newcastle upon Tyne

R J Cooke

KMcCormick

I J Griffin

J S Smith

S J Robinson

Regional Medical Physics Department

Freeman Hospital

Newcastle upon Tyne

D J Rawlings

K Faulkener

Nutrition Department Nutricia Ltd

Trowbridge

Wiltshire

J C K Wells

Correspondence to:

Dr R J Cooke

Ward 35, Leaze's Wing

Royal Victoria Infirmary

Newcastle upon Tyne

NE1 4LP

Email: R.J.Cooke@ncl.ac.uk

Accepted 14 November 1998
Keywords: body composition; dual emission energy $x$-ray absorptiometry; preterm

Measurement of body composition has important implications for nutritional support during infancy and childhood. Dual emission energy $x$-ray absorptiometry (DEXA) promised to provide a relatively easy, non-invasive, and safe method for the measurement of body composition during this time. Although the accuracy of DEXA has been questioned, ${ }^{12}$ recent data suggest that DEXA accurately measures lean mass (LM) and fat mass (FM). ${ }^{3}$ DEXA can also provide precise determinations of body composition. $^{4}$

Despite the critical inter-relation between early nutrition, growth, development, and subsequent health, ${ }^{5}$ there are few data on changes in body composition in preterm infants during the first year of life. This study therefore aimed to measure body composition using DEXA in preterm infants during the first year of life, and to compare the results to the term reference infant of Fomon. ${ }^{6}$

\section{Methods}

This prospective study began immediately before hospital discharge and lasted until 12 months corrected age. The study was approved by the Ethics Committee of the Newcastle and North Tyneside Health Authority and informed consent was obtained from the parent(s) or legal guardian.

Preterm infants with a gestational age of $\leqslant 34$ weeks and a birthweight of $\leqslant 1750 \mathrm{~g}$ were considered eligible for the study. Those without evidence of systemic disease, requiring no therapeutic intervention, and growing normally at the time of hospital discharge were enrolled. After hospital discharge all infants were formula fed and regular contact was maintained with the family throughout the study.

Infants were seen at the outpatient clinic every two weeks between discharge and term, monthly between term and 6 months, and at 9 and 12 months corrected age. At each clinic visit, weight, crown-heel length, and head circumference were measured. At term, 12 weeks, 6 months, and 12 months corrected age body composition was measured using DEXA (Hologic QDR 2000, Hologic Inc, Waltham, MA, USA). Scan analysis was performed using the Infant Whole Body Software (version 5.56).

Before each measurement, the scanning couch of the densitometer was moved to the starting position, and a radiation filter plate, provided by the manufacturer for use with infant whole body scans, was placed above the surface of the scanning couch. The scanning area was then covered with a commercial absorbent paper material and a calibration phantom (the " $T$ " bar) placed at one edge of the scanning area.

The naked infant was swaddled in a light cotton sheet and placed, either prone or supine, on the scanning surface parallel to the long axis of the couch, with the head positioned about $2 \mathrm{~cm}$ from the starting point of scan. Scans were performed without sedation but with a research nurse constantly in attendance. Scans were timed to begin when the infant was quiet. In the event of movement scans were not repeated. Anthropometric determinations were measured as described before. ${ }^{7}$

The data were analysed using a combination of paired $t$ tests, regression analysis, and analysis of variance. Post-hoc comparisons were determined using Fisher's test. Results were considered significant at $\mathrm{p}<0.05$. 


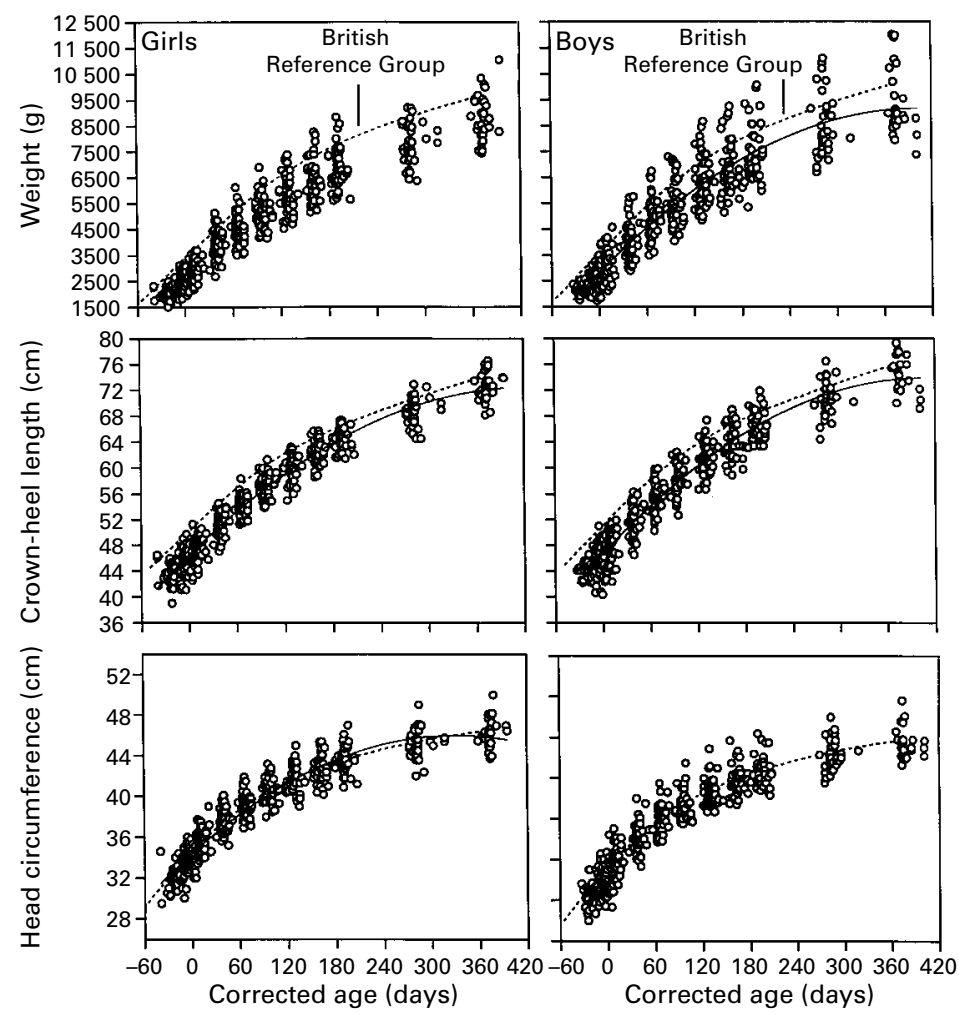

Figure 1 Growth data in study infants. Interrupted line represents 50th percentile for British reference standards in preterm infants. ${ }^{10}$
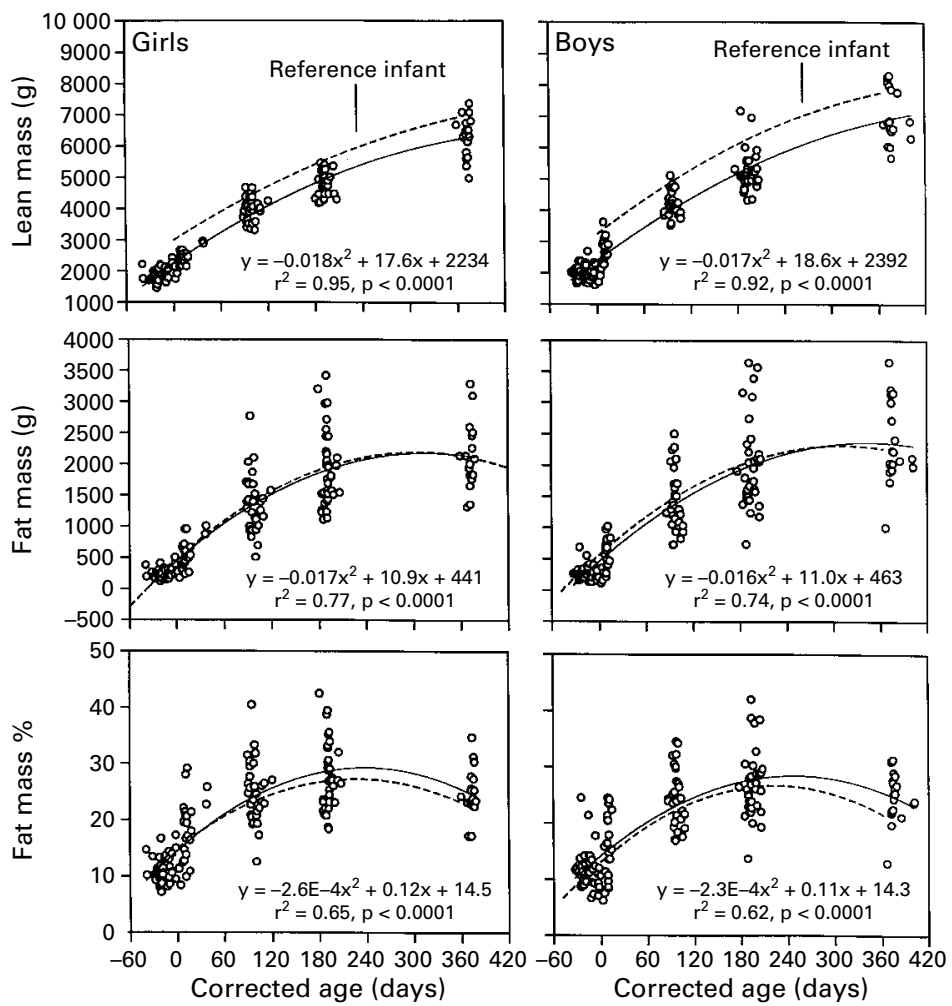

Figure 2 Body composition in study infants plotted as function of corrected age. Interrupted line represents the reference infant of Fomon. ${ }^{6}$

Data for the study infants were compared with the term reference infant of Fomon et $a l^{6}$ because the latter provides information not only on LM and FM but also on bone mineral mass (BMM). Values for LM and FM in the reference infant are also similar to that of de Bruin et al, who used total body electrical conductivity to measure body composition in term infants. $^{8}$

To examine whether bone mineral density (BMD) was influenced by body size, the approach of Prentice et al was used. ${ }^{9}$ The data were log transformed so that the regression coefficients provided information, in proportional terms, on the influence of each variable on BMM. Linear regression was initially performed between $\log \mathrm{BM}$ and log bone area (BA). A regression coefficient different from 1 indicated that BMD was not an appropriate correction for differences in BA. Stepwise regression was then performed to determine which factors affected the measurement of BMM.

\section{Results}

One hundred and twenty five infants were studied (mean (SD) birthweight $=1394$ (313) g, gestational age $=31.0$ (2.3) weeks). The anthropometric data of these infants were compared with British reference standards for growth in preterm infants (fig 1). ${ }^{10}$ The 50th percentile for the British reference group is represented by the interrupted line. In both sexes weight and crown-heel length were lower, while OFC was similar in the study when compared with that of the reference group.

DEXA derived body weight closely reflected that determined by conventional scales $(\mathrm{y}=$ $111+0.99 \mathrm{x}, \mathrm{r}^{2}=0.99, \mathrm{p}<0.0001$; where $\mathrm{y}=$ DEXA - weight, $\mathrm{x}=$ scale-weight, $\mathrm{n}=294$ measurements). The mean (SD) of the difference between DEXA derived and scale derived body weights were -37 and $307 \mathrm{~g}$, respectively ( $p=N S)$. The mean (SD) weights at discharge, term, 12 weeks, 6 months, and 12 months were 2114 (247) vs 2214 (310) g, 3069 (469) vs 3063 (515), 5402 (690) vs 5472 (779), 6976 (905) vs 6982 (992), and 8963 (1130) vs 8902 (1218) for scales and DEXA, respectively.

In girls and boys LM increased in a curvilinear manner with age $(p<0.0001)$ (fig 2$)$; the changes in LM between discharge and term (27 (4)) were significant by term $(\mathrm{p}<0.001)$. At discharge $(1910(232)<1993$ (222) g; p < $0.10)$, term 2377 (174); p < 0.01), 12 weeks (3958 (369) < 4208 (391); p 0.01), 6 months $(4842(369)<5225$ (595); $\mathrm{p}<0.005)$, and 12 months $(6308(638)<7000(814) ; \mathrm{p}<0.01)$ LM was less in girls than in boys.

FM and \%FM increased between discharge and 6 months corrected age (fig 2); the changes between discharge and term were also significant $(\mathrm{p}<0.001)$. Between 6 and 12 months, FM did not change but \% FM fell from 28 (6) to $25(4)$ in girls $(\mathrm{p}<0.05)$ and from $27(6)$ to 25 (4) in boys; $\mathrm{p}<0.05$ (fig 2). No significant differences were detected in FM or \% FM between the sexes. LM was less than the term reference infant at the same age while FM and $\% \mathrm{FM}$ of the study infants were similar to those of the term reference infant. ${ }^{6}$

Changes in body composition are expressed as a function of weight in fig 3 . In boys and girls LM and FM increased in a linear manner with 

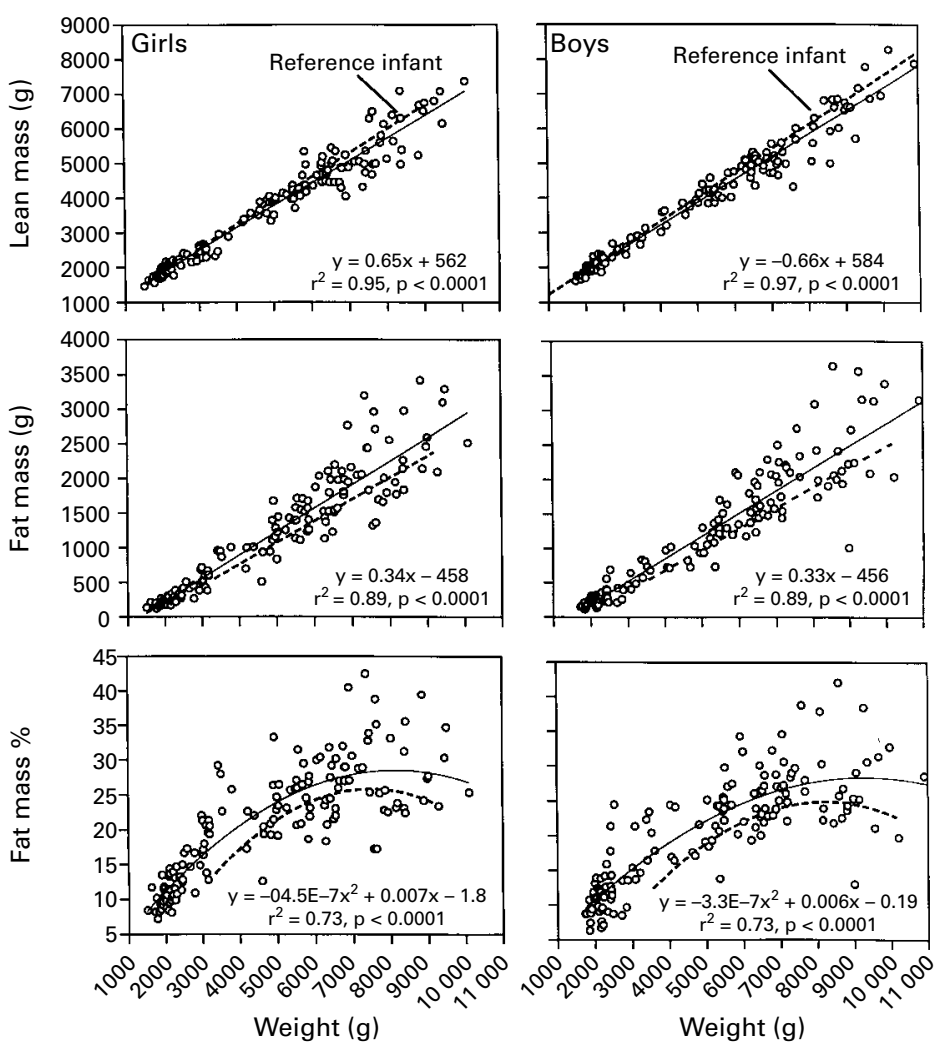

Figure 3 Body composition in study infants plotted as function of body weight. Interrupted line represents the reference infant of Fomon.

Table 1 Mean (SEM) changes in bone mass in preterm infants

\begin{tabular}{llllll}
\hline \multicolumn{2}{c}{ Discharge } & Term & 12 weeks & 6 months & 12 months \\
\hline Bone area $\left(\mathrm{cm}^{2}\right)$ & & & & \\
Boys & $203(24)$ & $268(51)$ & $403(47)$ & $494(62)$ & $641(72)$ \\
Girls $\quad 197(23)$ & $250(34)$ & $391(43)$ & $479(52)$ & $582(70)$ \\
Bone mineral mass $(\mathrm{g})$ & & & & \\
Boys & $28(4.7)$ & $44(13)$ & $87(19)$ & $129(28)$ & $189(33)$ \\
Girls & $27(5.2)$ & $43(14)$ & $82(17)$ & $122(23)$ & $171(31)$ \\
Bone mineral density $\left(\mathrm{g} / \mathrm{cm}^{2}\right)$ & & & & \\
Boys $\quad 0.14(0.01)$ & $0.17(0.04)$ & $0.21(0.02)$ & $0.25(0.03)$ & $0.29(0.03)$ \\
Girls & $0.13(0.01)$ & $0.16(0.02)$ & $0.21(0.02)$ & $0.26(0.03)$ & $0.29(0.03)$ \\
\hline
\end{tabular}

${ }^{\star}$ Boys $>$ girls $(\mathrm{p}<0.01)$.

weight (fig 3). At the same weight, no significant differences were detected in LM, $\mathrm{FM}$, or \%FM between girls and boys. At the same weight, LM was similar to that of the reference infant while $\mathrm{FM}$ and \% FM of the study infants were slightly greater. ${ }^{6}$

Table 1 gives the measurements of bone mass in girls and boys. BA, BMM, and BMD increased throughout the study; the changes in each variable were significant by term ( $p<0.01)$. Overall, boys had a greater BA and BMM than girls $(\mathrm{p}<0.01)$, largely reflecting differences at 12 months. No differences were noted in BMD between the sexes.

BMM was linearly related to BA $(y=-2.4+$ $1.67, \mathrm{r}^{2}=0.98, \mathrm{p}<0.0001$; where $\mathrm{y}=\log$ $\mathrm{BMM}$ and $\mathrm{x}=\log \mathrm{BA})$. However, the power coefficient, 1.67 (0.07), was greater than $1(\mathrm{p}<$ 0.0001 ), indicating that BMD had not totally corrected for differences in BA. Stepwise regression analysis indicated that, in addition to $\mathrm{BA}, \mathrm{BMM}$ determinations were significantly affected by body weight.

LM more closely correlated with crown-heel length $\left(r^{2}=0.96\right)$ than weight:length ratio $\left(r^{2}=\right.$
0.93). BMM was also closely related to crownheel length $\left(r^{2}=0.93\right)$.

\section{Discussion}

Data from this study indicate that DEXA accurately predicts body weight. However, accurate prediction of body weight does not mean accurate measurement of body composition. ${ }^{3}$ To assess in vivo accuracy of DEXA, data for the study infants were compared with those of the reference infant of Fomon. ${ }^{6}$ At the same age, LM was less, but FM and $\% \mathrm{FM}$ were similar to those of the reference infant. At the same weight, LM was identical with that of the reference infant, but FM and $\% \mathrm{FM}$ were slightly greater. This agrees with the recent observations which suggest that DEXA tends to overestimate FM. ${ }^{3}$

At term, 3, 6, and 12 months DEXA derived BMM in boys was 44, 87, 129, and 189 $\mathrm{g}$-values comparable with those published before. $^{311} 12$ However, DEXA measures total hydroxyapatite, which is $34 \%$ calcium, and DEXA derived body calcium values differ somewhat from the reference infant at the same weight; 16.7 vs $31.2,35.7$ vs 33.5, 47.8 vs 37.5, and 65.5 vs $47.4 \mathrm{~g}$ at term, 3, 6, and 12 months, respectively. These findings may be partly explained as follows.

DEXA derived BMM is affected by body size. ${ }^{1}$ When body size is small, as occurred at discharge, the skeleton is poorly mineralised and pixels containing small amounts of bone may be counted as LM which has an attenuation coefficient close to bone. ${ }^{2} \mathrm{BMM}$ and, therefore, calcium, may be underestimated.

DEXA derived BMM is affected by anteroposterior thickness of the body. ${ }^{13}$ If DEXA derived $\mathrm{BMM}$ increases with antero-posterior thickness, then changes in BMM may, in part, reflect the fourfold increase in body weight which occurred during this study. At 12 months, BMM and, therefore, total body calcium, might then be overestimated.

The accuracy of DEXA has been questioned, ${ }^{2}$ but precision is excellent. Mazess et al report a precision error of $1.4 \%, 1.0 \mathrm{~kg}$, and $0.8 \mathrm{~kg}$ for \%fat, fat mass, and lean body mass in healthy adults. ${ }^{4}$ Brunton et al report a coefficient of variation of $0.7 \%$ and $\sim 4.0 \%$ for lean and fat mass in piglets. ${ }^{3}$ Braillon et al report a mean precision of 1.2 and $2.4 \%$ in measuring femoral $\mathrm{BMC}$ in preterm infants and lumbar spine BMC in term infants, respectively. ${ }^{11}$

The changes between discharge and term in LM, FM, and BMM were $588(\mathrm{p}<0.0001)$, $287(\mathrm{p}<0.05)$, and $17.1 \mathrm{~g}(\mathrm{p}<0.001)$, respectively. For LM, net change was twice, for FM, net change just reached significance, while for $\mathrm{BMM}$, net change was $\sim 1.5$ times the critical difference needed to achieve significance. While whole body DEXA seems to measure change in mass relatively precisely, sample size determinations may vary depending on whether LM, FM, or BMM are the primary outcome variable.

It is assumed that BMM and BA change in a 1:1 manner. $\mathrm{BMD}$ is therefore considered an 
appropriate correction for bone size. Prentice $e t$ al suggest that this is not always true. ${ }^{9}$ They show that BMM in adults is also influenced by body size, and failure to include this in a regression model may lead to erroneous conclusions about the effects of interventionfor example, the effects of diet on bone mass. ${ }^{9}$ In this study changes in BMM were not directly proportional to those in BA but were significantly influenced by body size. As in adults, the validity of BMD as a reference for bone mass in epidemiological research in preterm infants must also be questioned.

The results of this study are important. Few data are available on the compositional nature or the effect of sex on growth in preterm infants. This study not only provides data on the nature of growth, but is the first to show differences between preterm boys and girls.

Debate exists about comparison of growth in preterm and term infants. Data from this study suggest that while preterm babies are smaller, the compositional nature of growth, in terms of LM and FM, may be similar to that of the term infant.

Whole body DEXA seems to measure LM and FM relatively accurately. However, controversy exists about the use of whole body DEXA for assessing bone mass. Molgaard et al suggest that $\mathrm{BMM}$ be directly related to $\mathrm{BA}^{14}$ Hannan et al suggest that this is not appropriate, because BA may be underestimated in conditions associated with reduced bone mass, such as anorexia nervosa. ${ }^{15}$ Data from this study suggest that changes in BMM are not proportional to those in BA and may be confounded by body size. Further studies are needed to determine the most appropriate way to express bone mass and the extent to which the clinical state may confound interpretation of DEXA derived values. Data from this study also suggest that LM and BMM might be reliably predicted from crown-heel length, but a larger sample size is needed before final conclusions can be drawn.

We thank the nursing and medical staff on the Special Care Baby Units of the Royal Victoria Infirmary and Ashington General Hospital for their advice and active support in performing this study.

This study was, in part, supported by a grant from Cow and Gate, Nutricia Ltd.

1 Brunton J, Bayley H S, Atkinson S A. Validation and application of dual-energy $\mathrm{x}$-ray absorptiometry to measure bone mass and body composition in small infants. $A m \mathcal{F}$ Clin Nutr 1993;58:839-45.

2 Roubenoff R, Kehayias JJ, Dawson-Hughes B, Heymsfield $\mathrm{SB}$. Use of dual-energy $\mathrm{x}$-ray absorptiometry in bodycomposition studies: Not yet a 'gold standard'. Am f Clin Nutr 1993;58:589-91.

3 Brunton J, Weiler HA, Atkinson SA. Improvement in the accuracy of dual energy x-ray absorptiometry for whole body and regional analysis of body composition: Validation using piglets and methodologic considerations in infants. Pediatr Res 1997;41:590-6.

4 Mazess RB, Barden HS, Bisek JP, Hanson J. Dual-energy $\mathrm{x}$-ray absorptiometry for total-body and regional bone-
mineral and soft-tissue composition. Am $\mathcal{f}$ Clin Nutr 1990;51:1106-12.

5 Barker DP. Mothers, babies, and disease in later life. London: BMJ Publishing Group, 1994.

6 Fomon S, Haschke F, Ziegler E, Nelson S. Body composition of reference children from birth to age 10 years. Am 7 Clin Nutr 1982;35:1169-75.

7 Cooke R, Perrin F, Moore J, Paule C, Ruckman K. Methodology of nutrient balance studies in the preterm infant. $\mathcal{F}$ ology of nutrient balance studies in the pre
Pediatr Gastroenterol Nutr 1988;7:434-40.

8 De Bruin NC, Van Velthoven KAM, De Ridder M, et al. Standards for total body fat and fat-free mass in infants. Arch Dis Child 1996;74:386-99.

9 Prentice A, Parsons T, Cole T. Uncritical use of bone mineral density in absorptiometry may lead to size-related artifacts in the identification of bone mineral determinants. Am f Clin Nutr 1994;60:837-42.

10 Freeman JV, Cole TJ, Chinn S, Jones PRM, White EM, Preece MA. Cross sectional stature and weight reference curves for the UK, 1990. Arch Dis Child 1995;73:17-24.

11 Braillon P, Salle B, Brunet J, Glorieux F, Delmas P, Meunier $P$. Dual energy x-ray absorptiometry measurement of bone mineral content in newborns: Validation of the technique. Pediatr Res 1992;32:77-80.

12 Molgaard C, Thomsen BL, Prentice A, Cole TJ, Michaelsen KF. Whole body bone mineral content in healthy children and adolescents. Arch Dis Child 1997;76:9-15.

13 Hannan WJ, Cowen SJ, Wrate RM, Barton J. Improved prediction of bone mineral content and density. Arch Dis Child 1995;72:147-9. 